\title{
Evaluation of Fibrosis and Histopathological Changes in the Psoas Muscle with E-Cadherin, Claudin-5 Expression and Demographic Data: An Autopsy Study
}

\author{
๑ Havva Erdem, • Hacer Yasar Teke*, ๑ Yusuf Sahin** \\ Ordu University Faculty of Medicine, Department of Medical Pathology, Ordu, Turkey \\ *Ordu University Faculty of Medicine, Department of Forensic Medicine, Ordu, Turkey \\ *Directorate of Forensic Medicine, Forensic Medicine, Ordu, Turkey
}

\section{Abstract}

Aim: It is known that muscle mass decreases with age. Sometimes it may be possible to adversely affect this reduction. It was aimed to investigate the contribution of claudin-5 and E-cadherin to this process.

Methods: Samples were taken from the psoas muscle of 55 cases autopsied for different reasons between 2018-2019. Age, gender, weight, height, chronic disease, and addiction were recorded. Histopathological degeneration parameters were evaluated. In addition, samples were prepared for immunohistochemical study. Evaluation for E-cadherin and claudin-5; no staining, weak staining, moderate staining and severe staining. Fibrosis was evaluated with Masson trichrome.

Results: There was a very strong and statistically significant inverse relationship between acidophilic sarcoplasma and staining with claudin-5 $(p<0.01)$. There was also a strong positive correlation between cellular fatty cell degeneration status and staining with claudin-5 ( $p=0.04)$. No correlation could be established between sociodemographic characteristics of the cases and staining with claudin-5 and E-cadherin, nor could there be a correlation between staining with E-cadherin and staining with claudin-5 ( $p>0.05$ ).

Conclusion: Claudin-5 can be a target protein that can be used in the detection and prophylaxis of degeneration and atrophy in striated muscles.

Keywords: Claudin-5, cadherins, socioeconomic factors, muscle, striated

\section{Introduction}

Muscle mass in humans is observed at maximum values at the ages of $25-30$ and decreases by about $25-30 \%$ by age 65 . This decrease in muscle mass is accompanied by an increase in non-contractile structures such as adipose tissue and connective tissue. In addition to the causes of age-related decrease in muscle mass, nutrition, sedentary lifestyle and chronic diseases can also affect this situation. The opposite situation may also have a protective effect (1).

The intersection complex consists of two main components [tigh junctions (TJ) and adherent junctions]. TJs are important in the epithelium in terms of barrier function and polarity. They are also involved in tissue differentiation and homeostasis (2). There is evidence that these functions are compromised, especially in older organisms, but these mechanisms have not been fully elucidated (3).

Claudins are the main transmembrane proteins of TJ. So far, 24 members of different species belonging to the claudin family have been identified $(2,4,5)$. Claudin proteins appear to be important for TJ formation and play an important role in controlling the paracellular permeability of ions. Indeed, Claudin gene expression is sufficient to induce the formation of TJ fibrils in fibroblast cells (6).

Address for Correspondence: Havva Erdem, Ordu University Faculty of Medicine, Department of Medical Pathology, Ordu, Turkey 
Cadherins constitute the most basic component of adhesive junctions. Cadherins are associated with the intercellular connection and the cytoskeletal system with the help of the catenin family (7). It has been observed that different expression patterns and dynamic changes of cadherins during development affect the function of cadherin in various morphogenetic events (8).

In this study, it was aimed to reveal the expression differences of claudin-5 and E-cadherin which are components of the junction complex, in the psoas muscle and the relation of these differences with the histopathological changes in the muscle with aging, fibrosis, adipose tissue as well as demographic data.

\section{Methods \\ Study Design}

This study was ethically approved by the Ordu University clinical research Ethics Committee (217/2020). In addition, the material in the study was autopsy samples and necessary legal and official permissions were obtained.

In this study, samples were taken from the psoas muscle of 55 cases autopsied for different reasons between 2018 and 2019. Simultaneous excel record files were created by selecting appropriate autopsies between 2018-2019. Age, gender, weight, height, chronic disease, and addiction were recorded. Histopathological fatty cell degeneration, fibrosis, acidophilic sarcoplasma, streaking, vacuolization, disruption in fibers, fragmentation, cellular infiltration, lipofuscin pigment accumulation indicating tissue degeneration and injury were evaluated (8-10).

Paraffin blocks were prepared using the manual microarray method from existing samples. Five $\mu \mathrm{m}$ thick sections were taken from these muscle samples. Sections were stained with $\mathrm{H} \& \mathrm{E}$ stain. The preparations were evaluated under light microscopy (Nikon eclipse $\mathrm{Ni}-\mathrm{U}$ Tokyo/Japan) at different magnifications. Histopathological evaluation was graded as 1/0 for each parameter [Figure 1. (A-E)] (8-10).

In addition, the scores of these parameters were summed up and the degeneration score was obtained. The average score was obtained according to age groups. The score belonging to the degeneration was evaluated as $1 / 0$. The score for claudin-5 and E-cadherin were evaluated as semiquantitatively $(11,12)$.

\section{Immunohistochemical Study}

Samples were taken from the tissues and then sections with a thickness of $5 \mu$ were taken on the poly- laminated slide. Prepared for immunohistochemical (IHC) study. A Leica Bond-Max IHC staining device (Vision Biosystems, Melbourne, Australia) was used for the IHC study. It was stained with claudin-5 (Epitomics (AC-0212A), $0.1 \mathrm{~mL}$ (1: 100). Slides were evaluated with a light microscope. Cytoplasmic membrane staining was considered positive.

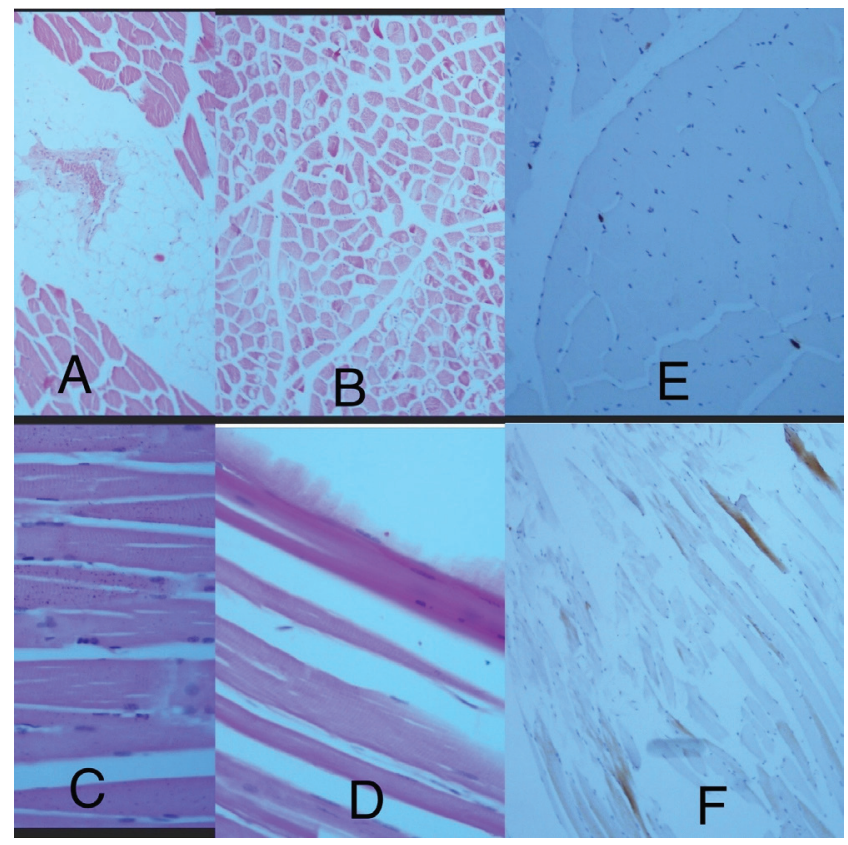

Figure 1. (A-E): A- Fat cell accumulation in muscle tissue (fatty degeneration) (H\&EX100), B- Vacuolization and disruption, fragmentation in muscle tissue (H\&EX40), C- Deposition of lipofuscin pigment in muscle tissue (H\&EX400), D- Acidophiliclike changes in muscle tissue (H\&EX400), E-claudin-5 expression in muscle tissue (grade 1) (x100), F-claudin-5 expression in muscle tissue (grade 2) (x100)

Four grades were evaluated. 0 staining (no staining), 1+ (weak) (1\% to $10 \%)$ staining, $2+$ (moderate) staining $(11 \%$ to $50 \%), 3+$ (severe) staining (over 50\%) were evaluated $(11,12)$.

E-cadherin [mouse monoclonal antihuman antibody, Biogenex (10 microliters diluted per $1 \mathrm{~mL}$ )] was applied. The stained slides were examined with a Nikon Eclipse Niu microscope and photographs were taken. Grading of IHC slides was done semiquantitatively.

Evaluation for E-cadherin; 0 grading (no staining), 1+ (weak) (1\% to $10 \%$ staining), $2+$ (moderate) (11\% to $50 \%$ staining), $3+$ (severe) (over $50 \%$ staining) were evaluated $(11,12)$.

\section{Histochemical Study}

Masson's Trichrome Stain kit was used to evaluate fibrosis. The presence of fibrosis was evaluated.

\section{Statistical Analysis}

The data were collected and analyzed using Statistical Package for software. In addition to descriptive statistics, the chi-square test was used in classifying categorical data. Spearman Correlation analysis was performed to investigate the relationship between staining and sociodemographic data and histopathology findings. In addition, the quality of the relationship was revealed by performing multiple regression analyses. In all types of analyzes, a 5.0\% significance level was used. 


\begin{tabular}{|c|c|c|c|c|c|}
\hline & BMI $<18.5$ & $18.6<\mathrm{BMI}<24.9$ & $25.0<\mathrm{BMI}<29.9$ & $30.0<$ BMI $<39.9$ & $p$ \\
\hline & n (\%) & n (\%) & n (\%) & n (\%) & \\
\hline \multicolumn{5}{|l|}{ Gender } & $p=0.46$ \\
\hline Female & - & $1(\% 1.81)$ & $3(5.45)$ & $2(3.63)$ & - \\
\hline Male & $4(7.27)$ & $19(34.54)$ & $19(34.54)$ & $7(12.72)$ & - \\
\hline \multicolumn{5}{|l|}{ Age } & $p=0.86$ \\
\hline $35-65$ & $2(3.63)$ & $12(21.81)$ & $16(29.09)$ & $7(12.72)$ & - \\
\hline $66-85$ & $2(3.63)$ & $7(12.72)$ & $5(9.09)$ & $2(3.63)$ & - \\
\hline 85 and above & - & $1(1.81 \%)$ & $1(1.81 \%)$ & - & - \\
\hline \multicolumn{5}{|l|}{ Alcohol } & $p=0.62$ \\
\hline Using & $3(5.45)$ & $3(5.45)$ & $4(7.27)$ & $2(3.63)$ & - \\
\hline Not using & $1(1.81)$ & $17(30.90)$ & $18(32.72)$ & $7(12.72)$ & - \\
\hline \multicolumn{5}{|l|}{ Cigarette } & $p=0.16$ \\
\hline Using & $4(7.27)$ & $15(27.27)$ & $13(23.63)$ & $4(7.27)$ & - \\
\hline Not using & - & $5(9.09)$ & $9(16.36)$ & $5(9.09)$ & - \\
\hline \multicolumn{5}{|l|}{ Disease state } & $p=0.67$ \\
\hline No known disease & $1(1.81)$ & $7(12.72)$ & $8(14.54)$ & $2(3.63)$ & - \\
\hline CAD & $3(5.45)$ & $10(18.18)$ & $10(18.18)$ & $6(10.90)$ & \\
\hline CAD and COPD & - & $1(1.81)$ & $1(1.81)$ & - & - \\
\hline Psychiatric disease & - & - & $3(5.45)$ & - & - \\
\hline
\end{tabular}

Table 2. Results of multiple regression analysis of factors associated with claudin staining

\begin{tabular}{|c|c|c|c|c|c|c|}
\hline & $\beta$ & S.E & $p$ & OR & \multicolumn{2}{|c|}{$95 \% \mathrm{Cl}$} \\
\hline & & & & & Lower & Upper \\
\hline Y & -1.35 & 0.66 & 0.04 & 0.25 & 0.07 & 0.94 \\
\hline A & 2.04 & 0.72 & $p<0.01$ & 7.73 & 1.87 & 31.87 \\
\hline
\end{tabular}

\section{Results}

$89.1 \%(n=49)$ of 55 patients were male and $10.9 \%$ $(n=6)$ were female. The mean age of the cases was $60.67 \pm 13.38$ and the youngest case was 35 and the oldest case was 90 years old. The sociodemographic data obtained from the cases according to their body mass index (BMI) are presented in Table 1.

As a result of staining the muscle samples of the cases with E-cadherin, $92.7 \%(n=51)$ of the samples were not stained, but only $7.3 \%(n=4)$ were stained. Staining the psoas muscle samples of 55 cases with claudin- $5,29.1 \%$ $(n=16)$ were not stained, and $70.9 \%(n=39)$ were stained. Five $(9.1 \%)$ of the stained samples were strongly stained with claudin-5.

No correlation could be established between sociodemographic characteristics of the cases and staining with claudin-5 and E-cadherin, nor could there be a correlation between staining with E-cadherin and staining with claudin-5 ( $p>0.05)$. In addition, there was no correlation between E-cadherin staining and histopathological features ( $p>0.05)$.

In the correlation analysis made to evaluate the relationship between histopathological findings (Graph 1) and staining (Graph 2); It was observed that there was a very strong and statistically significant inverse relationship between acidophilic sarcoplasma and staining with claudin- 5 $(r=-0.41, p<0.01)$. There was also a strong positive correlation between cellular fatty cell degeneration status and staining with claudin-5 $(r=0.28, p<0.05)$ (Graph 2$)$; Staining with claudin-5 in non-fatty cells is statistically significant 0.25 times higher. In addition, staining with claudin-5 in cells with acidophilic sarcoplasma is statistically significantly higher than 7.73 times. The relationship between histopathological findings and age groups was demonstrated by Graph 1, and when age groups were grouped as 35-45, 46-55, 56-65, 66 and above, it was observed that degeneration increased as age progressed. The same relationship with claudin-5 is shown in Table 2,3.

\section{Discussion}

There are many publications stating that myofibrillary decline starts around the age of 25 and the rates of this 
Erdem et al. Cadherin, Claudin-5 Expression of Muscle

Table 3. Relationship between age groups and degeneration score, claudin score, chronic disease

\begin{tabular}{|c|c|c|c|c|c|c|c|}
\hline Age & & $35-45$ & $46-55$ & $56-65$ & $66-90$ & 65 age below & 66 age above \\
\hline Degeneration score & \multirow{3}{*}{ 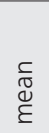 } & 3.5 & 3.57 & 3.52 & 4 & 3.56 & 4 \\
\hline Claudin score & & 0.8 & 1 & 0.75 & 0.77 & 0.81 & 0.77 \\
\hline Chronic disease & & 0.2 & 0.57 & 0.85 & 0.94 & 0.62 & 0.94 \\
\hline
\end{tabular}

decline increase with age. In some of these publications, it has been noted that the decrease between the ages of 3050 is approximately $15 \%$ and after this age it is 30\% every ten years. In addition, there are determining factors such as changes in the nervous system with age, changes in the structure and function of the neuromuscular junction, fat infiltration, cellular and molecular modifications of the muscle. Again, lipofuscin (age-related pigment) and fat deposit into the muscle and promote muscle tissue loss. This ensures less tone and ability to contract $(9,10)$. Although adult skeletal muscle is fully differentiated, fibers retain the ability to regenerate and replace it in response to an injury. However, in pathological conditions or during the aging process, functional muscle damage may result in the formation of fibrotic tissue regeneration $(9,10)$.

One of the parameters evaluated in this study was the association of psoas muscle fibrosis with age and chronic diseases. In this evaluation, age was evaluated individually as well as in age groups. They had chronic cardiovascular disease, allergy and psychiatric diseases in terms of chronic diseases. The other two diseases were excluded from the group and their relationship with the more common chronic cardiovascular disease was examined. It was observed that this disease increased with age, and fibrosis increased with age but it was not statistically significant. This is probably because the sample is small.

Atrophy and degeneration often occur for different reasons. They may occur due to mechanical reasons or immobility. Degeneration can also occur by many factors such as inflammation, abnormal mechanical strength, and altered vascularization (13).

In animal models of muscle injuries, atrophy characterized by fat accumulation has been found to be effective in early and middle-stage diseases. It has also been shown that high levels of inflammation exhibit an active cycle of degeneration and regeneration. In this study by Gibbons et al. (10) muscle loss was so severe that in the vast majority of samples, muscle tissue was reported to be replaced by an irregular, vascular connective tissue network with high macrophage density. It has been stated that in clinical imaging of such tissues, this appearance resembles a muscle, which may prevent the detection of true loss (10).

Although there is no muscle injury known in this study, parameters such as BMI as well as age and chronic diseases are associated with degeneration and atrophy.
In this study, it was observed that fatty degeneration, histopathological signs of degeneration and chronic diseases tended to increase with age, and this trend was accompanied by loss of claudin-5 (Table 3 ).

Inflammation is prominent in an increasing number of disease states where changes in TJ have been observed. Changes in TJs has been associated with inflammatory diseases associated with many autoimmune mechanisms such as collagenous colitis, psoriasis, multiple sclerosis/ encephalomyelitis, Crohn's disease, ulcerative colitis and arthritis. In addition, proinflammatory cytokines have also been shown to induce apoptosis at the cell level and affect permeability by affecting the structure of TJs (13).

In a study on changes in claudin expression profiles, it was reported that it contributed to epithelial lung dysfunction during infection and inflammation (14).

It has been reported in the literature that the change in claudin levels contributes to the disruption of the blood barrier $(15,16)$. In several diseases related to barrier dysfunction, various pathologies of the nervous system characterized by a pronounced neuroinflammatory component such as Alzheimer's disease, multiple sclerosis, diabetic retinopathy and retinopathy of prematurity, the dysfunction of the brain and retinal barriers contributes to the pathogenesis of these diseases and even to the pathogenesis of these diseases (17).

Recent reports have also shown that claudin- 5 is reduced in retinal endothelial cells due to endoplasmic reticulum stress, which also plays a role in vascular dysfunction in diabetic retinopathy (17).

In this study, a statistical relationship was not found when evaluated in terms of chronic diseases. When it is divided into age groups and evaluated, it has been observed that it tends to decrease (muscle mass). Since the youngest adult case was 35 years old, no comparison was made with muscle tissue under 35 years old. An evaluation was made with claudin-5 in the form of muscle expression, and the blood level was not measured. However, when the degeneration score was compared with the claudin score, an inverse relationship was observed. No relationship was found between E-cadherin and claudin-5. The reason for this is that E-cadherin is mostly in epithelial localization and claudin-5 is in endothelial localization, and therefore, it can be difficult to show this relationship in the striated muscle. This situation constitutes the limitation of our study. 
In previous references, the increase in fat tissue and fibrosis in muscle tissue with age is indirectly indicative of atrophy. It can also indicate degeneration $(13,14,17)$. In this study, an inverse relationship was observed between the increase in adipose tissue and claudin-5, and it was observed that the increase in adipose tissue increased with age. Based on this result, we can say that atrophy and degeneration occur with age, and as a result, claudin expression decreases. Although a positive correlation was observed between acidophilic sarcoplasma and claudin-5, it was seen that this parameter showed a homogeneous distribution when the age distribution was examined. When the degrees of degeneration including acidophilic sarcoplasma were scored, we observed that claudin expression decreased with age and the score of the degeneration increased.

In a study by Ozawa (18), it was shown that expression of the DsRed-labeled E cytoplasmic domain in C2C12 myoblasts inhibits myoblast fusion as well as the transport of endogenous cadherins, including $\mathrm{N}$-cadherin and M-cadherin (18).

Hollnagel et al. (19) in their study, analyzed the known components of adhesive plaques on Western blot of proteins from regenerated muscle to investigate alternative molecules that could mediate cell adhesion in the absence of M-cadherin. Interestingly, heterozygous and homozygous animals reported that they contained levels of $\mathrm{N}$ - and $\mathrm{E}$-cadherin, both of which were regulated by a significant increase during degeneration (19).

In this study, since the expression of E-cadherin was very low, no relationship was found with age groups when evaluated in terms of the trend in expression of E-cadherin. Likewise, no relationship was found with histomorphological findings. As stated above, the very low expression level could possibly be the cause.

\section{Study Limitations}

Causes of death such as drowning or gas poisoning were not included in this study. Again, those with a color change on the corpse were not included in the study. The sampled muscles were chosen from areas that could be hidden in order not to disturb the integrity of the body. These reasons are the limitations of the study.

\section{Conclusion}

As a result, claudin-5 tends to decrease in striated muscle tissue in degeneration and atrophy. Therefore, it can be a target protein that can be used in the detection and prophylaxis of degeneration and atrophy in striated muscles. No relationship was found between E-cadherin and striated muscle degeneration.

\section{Authorship Contributions}

Concept: H.E., Design: H.E., H.Y.T., Y.S., Data Collection or Processing: H.E., H.Y.T., Analysis or Interpretation: H.E.,
H.Y.T., Y.S., Literature Search: H.E., Y.S., Writing: H.E., H.Y.T.

Conflict of Interest: No conflict of interest was declared by the authors.

Financial Disclosure: The authors declared that this study received no financial support.

\section{References}

1. Thompson LV. Skeletal muscle adaptations with age, inactivity, and therapeutic exercise. J Orthop Sports Phys Ther 2002;32:44-57.

2. González-Mariscal L, Betanzos A, Nava P, Jaramillo BE. Tight junction proteins. Prog Biophys Mol Biol 2003;81:1-44.

3. D'Souza T, Sherman-Baust CA, Poosala S, Mullin JM, Morin PJ. Age-related changes of claudin expression in mouse liver, kidney, and pancreas. Journals of Gerontology Series A: Biomedical Sciences and Medical Sciences 2009:64:1146-53.

4. Tsukita S, Furuse M, Itoh M. Multifunctional strands in tight junctions. Nat Rev Mol Cell Biol 2001;2:285-93.

5. Förster C. Tight junctions and the modulation of barrier function in disease. Histochem Cell Biol 2008;130:55-70.

6. Furuse M, Sasaki H, Fujimoto K, Tsukita S. A single gene product, claudin-1 or -2 , reconstitutes tight junction strands and recruits occluding in fibroblasts. J Cell Biol 1998;143:391-401.

7. Redfield A, Nieman MT, Knudsen KA. Cadherins promote skeletal muscle differentiation in three-dimensional cultures. J Cell Biol 1997;138:1323-31.

8. Hafez MS, Makhlouf NA. Histological changes in sarcopenia and the possible protective role of angiotensin-converting enzyme inhibitors in male albino rats. The Egyptian Journal of Histology 2011;34:762-77.

9. Ríos IDP. Loss of Muscle Mass Induced by Aging. Rev Cienc Salud Bogotá Colombia 2019;17:223-4.

10. Gibbons MC, Singh A, Anakwenze $O$, et al. Histological Evidence of Muscle Degeneration in Advanced Human Rotator Cuff Disease. J Bone Joint Surg Am 2017;99:190-9.

11. Erdem H, Canakcı E, Karatas A, Akcay Celik M, Kilinc A. Evaluation of Distant Organ Effect of Renal Ischemia and Reperfusion with Claudin-5. J Crit Intensive Care 2020;11:3-7.

12. Sahin A, Erdem H, Akçay Çelik M, Cankaya S, Aslan Ali. Correlation of E-cadherin/Beta-Catenin Expression with Localization in Squamous Epithelial Cell Carcinoma and Basal Cell Carcinoma. Online Turkish Journal of Health Sciences 2020;5:464-73.

13. Skrovanek S, Valenzano MC, Mullin JM. Restriction of sulfurcontaining amino acids alters claudin composition and improves tight junction barrier function. Am J Physiol Regul Integr Comp Physiol 2007;293:R1046-55.

14. Van Itallie CM, Anderson JM. The role of claudins in determining paracellular charge selectivity. Proc Am Thorac Soc 2004; 1:38-41. 
15. Huang $M$, Chen SP, Dai $Y Y$, et al. [Cathepsin $L$ mediates glomerular endothelial cell injury by cleavaging complement C3 in trichloroethylene-sensitized mice]. Zhonghua Lao Dong Wei Sheng Zhi Ye Bing Za Zhi. 2021;39:5-11.

16. Zhang S, Wang $X$, Cheng F, et al. Network PharmacologyBased Approach to Revealing Biological Mechanisms of Qingkailing Injection against IschemicStroke: Focusing on Blood-Brain Barrier. Evid Based Complement Alternat Med 2020;2020:2914579.

17. Gonçalves A, Ambrósio AF, Fernandes R. Regulation of claudins in blood-tissue barriers under physiological and pathological states. Tissue Barriers 2013;1:e24782.
18. Ozawa M. E-cadherin cytoplasmic domain inhibits cell surface localization of endogenous cadherins and fusion of C2C12 myoblasts. Biol Open 2015;4:1427-35.

19. Hollnagel A, Grund C, Franke WW, Arnold HH. The cell adhesion molecule M-cadherin is not essential for muscle development and regeneration. Mol Cell Biol 2002;22:476070. 